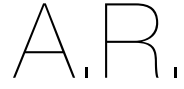

ARTIGO DE REVISÃO

1 Escola Superior de Tecnologia da Saúde de Coimbra do Instituto Politécnico de Coimbra,

Rua 5 de Outubro - SM Bispo, Apartado 7006, 3046-854 Coimbra Portugal

2 Departamento de Dietética e Nutrição, Escola Superior de Tecnologia da Saúde de Coimbra do Instituto Politécnico de Coimbra,

Rua 5 de Outubro - SM Bispo, Apartado 7006, 3046-854 Coimbra,

Portugal

Endereço para correspondência: Beatriz Lúcio Rua São Sebastião, n.’ 27. Campelos, Portugal beatrizsslucio@gmail.com

Histórico do artigo:

Recebido a 14 de maio de 2020 Aceite a 28 de setembro de 2020

\title{
ARSÉNIO: UM POTENCIAL TÓXICO PARA O CONSUMIDOR DAS ALGAS NORI?
}

\author{
ARSENIC: A TOXIC POTENTIAL OF NORI SEAWEED?
}

Beatriz Lúcio'; ${ }^{1 ;}$ Eduarda Carvalho'; Joana Fernandes'; Rafaela Melo'; Ana Lúcia Baltazar²

\section{RESUMO}

As algas são uma fonte de novos compostos bioativos, como alguns polissacarídeos, que não são encontrados em plantas terrestres, que podem conferir propriedades benéficas para a saúde. Segundo os autores Desideri, Mac Minagail e Zhao YF sabe-se que as algas marinhas contêm altos níveis de arsénio, em diferentes formas químicas.

O principal objetivo desta revisão da literatura é avaliar os níveis de toxicidade do arsénio, isto é, a quantidade mínima que induz uma reação adversa na saúde do consumidor da espécie de algas Porphyra.

A elaboração deste artigo foi feita com base numa revisão da literatura nas bases de pesquisa científica "Science Direct" e "Google Académico".

O arsénio inorgânico, que apresenta toxicidade, foi detetado em concentrações elevadas apenas nas algas hijiki (Sargassum fusiforme), sendo que as algas Nori não apresentaram valores significativos deste elemento (19,2 \pm 8,4 mg/kg).

Conclui-se que nas algas Nori, quando consumidas com moderação (não excedendo as 12 g/dia), a concentração de arsénio inorgânico não é muito elevada, pelo que não é considerada tóxica para os consumidores.

\section{PALAVRAS-CHAVE}

Algas Nori, Arsénio, Arsénio inorgânico, Saúde, Toxicidade

\section{ABSTRACT}

Seaweed is a source of new bioactive compounds, such as some polysaccharides, which are not found in terrestrial plants and can confer beneficial health properties. According to the authors Desideri, Mac Minagail and Zhao YF, it is known to contain high levels of arsenic in different chemical forms.

The main aim of this literature review is to assess the levels of toxicity of arsenic, that is, the minimum amount that induces an adverse reaction on the health of the consumer of the Porphyra algae species.

The preparation of this article was base on a literature review on the scientific research bases "Science Direct" and "Google Scholar". Inorganic arsenic, which has toxicity, was detected in high concentrations only in hijiki algae (Sargassum fusiforme), and Nori did not present significant values for this elemento $(19.2 \pm 8.4 \mathrm{mg} / \mathrm{kg})$.

Nori algae when consumed in moderation (not exceeding $12 \mathrm{~g} /$ day) the concentration of inorganic arsenic is not very high, so it is not considered toxic for consumers.

KEYWORDS

Seaweed Nori, Arsenic, Inorganic arsenic, Health, Toxicity

\section{INTRODUÇÃo}

As algas marinhas são frutos do mar consumidos maioritariamente nos países asiáticos (1). Na Europa e em muitos países ocidentais, o uso de algas para fins cosméticos e médicos aumentou recentemente (2). As algas são uma fonte de novos compostos bioativos, como alguns polissacarídeos (polissacarídeos estruturais da parede celular, fucoidanas, xilanas, ulvanas, de armazenamento) (3), que não são encontrados em plantas terrestres, que podem conferir propriedades benéficas para a saúde, nomeadamente no controlo de peso, síndrome metabólica, diabetes tipo II e doenças do trato digestivo $(4,5)$.
A par do crescente consumo das algas processadas, a segurança tornou-se uma preocupação (6) uma vez que são uma importante fonte de exposição a elementos não essenciais ou tóxicos, como o cádmio (Cd), mercúrio $(\mathrm{Hg})$, chumbo $(\mathrm{Pb})$, antimónio (Sb) e arsénio (As) (7). Estes elementos são componentes naturais da crosta terrestre e dos sistemas biológicos pelo que a capacidade das algas para acumular metais pesados depende da biodisponibilidade destes na água circundante $(2,8,9)$. Sabe-se que as algas geralmente consumidas podem conter altos níveis de arsénio, sob diferentes formas, espécies de arsénio inorgânico, como As (III) ou As (V) (10), 
ou arsénio orgânico, como arsenobetaína e arseno-açúcares (AsSug) (11). O arsénio inorgânico não é benéfico para a saúde, uma vez que produz carcinogénese do fígado, rim, bexiga e pele e, portanto, a sua concentração na dieta deve ser o mais baixa possível (11, 12). A maioria dos organismos aquáticos tem a capacidade de metabolizar as formas inorgânicas, para as menos tóxicas tais como dimetilarsinato (DMA) e arsenobetaína $(13,14)$. No entanto, certas algas não usam esse processo de conversão metabólica e passam a ter proporções significativas de formas inorgânicas (13, 15-17).

Este estudo incidiu numa cultura de algas específica, a Porphyra, popularmente conhecida como Nori. Esta possui um teor de proteína equiparável a leguminosas, vitamina $\mathrm{C}$, minerais, oligoelementos, bem como polissacarídeos bioativos (porfirina), polifenóis, clorofila e carotenóides (18-20).

Dada a importância desta temática, o principal objetivo desta revisão da literatura é avaliar os níveis de toxicidade do arsénio nas algas Nori, para os consumidores. Neste sentido, é fulcral avaliar a concentração de arsénio nas algas Nori.

\section{METODOLOGIA}

A elaboração deste artigo foi feita com base numa revisão da literatura nas bases de pesquisa científica "Science Direct" e "Google Académico". Cruzaram-se as palavras-chave: "seaweed Nori", "toxicity", "health" e "inorganic arsenic", obtendo um total de 356 artigos datados de 2000 até ao presente. Apesar deste critério, abriram-se duas exceções, considerando artigos de 1993 e 1988, uma vez que estes demonstraram ter informações cruciais para a elaboração deste trabalho.

Selecionaram-se 48 artigos a partir da leitura dos títulos e dos resumos, considerando aqueles que avaliavam a concentração de arsénio nas algas Nori e o seu nível de toxicidade para os consumidores. Após a leitura na íntegra dos mesmos, utilizaram-se 26 artigos para a elaboração desta revisão, de acordo com a sua pertinência para a temática.

\section{Constituição das Algas Nori}

As algas Nori apresentam uma elevada concentração de minerais na sua constituição. A nível nutricional esta alga é fonte hidratos de carbono, proteínas, fibra, vitamina C, minerais, polissacarídeos bioativos, polifenóis, clorofila e carotenoides e ácidos gordos (18) (Tabela 1).

\section{Tabela 1}

Constituição das Algas Nori

\begin{tabular}{lc} 
MINERAIS & $\begin{array}{c}\text { CONCENTRAÇÃO } \\
\text { (POR } 100 \mathrm{~g} \text { DE PESO) }\end{array}$ \\
\hline Cálcio & $34,2 \mathrm{mg}$ \\
\hline Potássio & $301,2 \mathrm{mg}$ \\
\hline Magnésio & $108,3 \mathrm{mg}$ \\
\hline Sódio & $119,7 \mathrm{mg}$ \\
\hline Cobre & $0,1 \mathrm{mg}$ \\
\hline Ferro & $5,2 \mathrm{mg}$ \\
\hline lodo & $1,3 \mathrm{mg}$ \\
\hline Zinco & $0,7 \mathrm{mg}$ \\
\hline
\end{tabular}

\section{Arsénio nas Algas Marinhas}

Relativamente à concentração de arsénio, esta varia consoante os diferentes locais onde as algas Nori se desenvolvem $(11,21)$, pelo que, foram realizados diferentes estudos para determinar esta concentração. Sartal et al. determinaram $43,7 \pm 0,8 \mathrm{mg} / \mathrm{kg}$ de arsénio total (16) e Almela et al. $23,7 \pm 0,5 \mathrm{mg} / \mathrm{kg}$ de peso seco de arsénio total para a alga Nori (22). O conteúdo total de arsénio descrito na bibliografia para algas comestíveis varia entre 2,0 e 172 mg/kg (22). Os produtos de algas castanhas (hijiki, kombu, wakame, arame) apresentaram concentrações mais altas de arsénio do que as algas vermelhas (como a Nori) (23), que apresenta $19,2 \pm 8,4 \mathrm{mg} / \mathrm{kg}$, sendo que a hijiki mantém as concentrações mais altas (83,7 mg/kg) (15). Assim, o consumo de $3 \mathrm{~g}$ de algas por dia

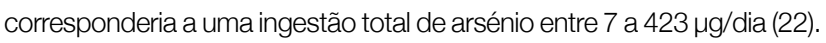
O arsénio inorgânico, que apresenta toxicidade, foi detetado em concentrações elevadas apenas nas algas hijiki, sendo que as algas Nori não apresentaram valores significativo deste elemento (13). Almela et al. determinaram $0,57 \pm 0,04 \mathrm{mg} / \mathrm{kg}$ de peso seco de arsénio inorgânico para a alga Nori (22) (Tabela 2).

De acordo com a Organização Mundial da Saúde (OMS), a ingestão diária tolerável de arsénio inorgânico, para um peso corporal de $68 \mathrm{~kg}$, é $0,146 \mathrm{mg} / \mathrm{dia}(24,25)$. Neste sentido, tanto o consumo médio (3 g/ dia) como o consumo máximo (12 g/dia) de alga Nori dão origem a níveis de ingestão inorgânica inferiores à ingestão diária tolerável (22). Apesar da alga Nori ser consumida maioritariamente em cru, a quantidade de arsénio total diminui durante o processo de cozimento (16).

Tabela 2

Constituição das Algas Nori

\begin{tabular}{|c|c|c|}
\hline & ALGA NORI & INGESTÃO DIÁRIA \\
\hline $\begin{array}{l}\text { Concentração } \\
\text { total de arsénio }\end{array}$ & $\begin{array}{l}43,7 \pm 0,8 \mathrm{mg} / \mathrm{kg}(16) \\
23,7 \pm 0,5 \mathrm{mg} / \mathrm{kg}(22) \\
19,2 \pm 8,4 \mathrm{mg} / \mathrm{kg}(15)\end{array}$ & $\begin{array}{c}\text { O consumo de } 3 \mathrm{~g} \text { de algas } \\
\text { comestíveis por dia corresponde } \\
\text { a uma ingestão total de arsénio } \\
\text { entre } 7 \text { a } 423 \mu \mathrm{g} / \mathrm{dia} \text { (22) }\end{array}$ \\
\hline $\begin{array}{l}\text { Concentração } \\
\text { de arsénio } \\
\text { inorgânico }\end{array}$ & $0,57 \pm 0,04 \mathrm{mg} / \mathrm{kg}(22)$ & $\begin{array}{l}\text { Tanto o consumo médio (3 g/dia) } \\
\text { como o consumo máximo } \\
\text { (12 g/dia) de alga Nori dão origem } \\
\text { a níveis de ingestão inorgânica } \\
\text { inferiores à ingestão diária } \\
\text { tolerável definida pela OMS (22) }\end{array}$ \\
\hline
\end{tabular}

\section{Legislação Sobre o Uso de Algas Marinhas para Consumo Humano}

Na Comunidade Europeia, o Regulamento (CE) n. ${ }^{\circ} 466 / 2001$, de 8 de março de 2001, estipula o teor máximo de certos contaminantes nos produtos alimentícios, no entanto não inclui as algas.

França foi o primeiro país europeu a estabelecer regulamentos sobre o uso de algas marinhas para consumo humano como alimentos não tradicionais. Atualmente, doze macroalgas (seis algas castanhas, cinco algas vermelhas, duas algas verdes) e duas microalgas são autorizadas. Essas algas devem atender às normas de segurança em termos de critérios toxicológicos e bacteriológicos $(2,3,22,26)$.

\section{ANÁLISE CRÍTICA}

Com o crescente consumo de algas marinhas, nomeadamente as Nori, revelou-se ser necessário a realização de mais estudos sobre a constituição da mesma, para garantir a segurança dos consumidores. Do ponto de vista nutricional estas algas são consideradas interessantes, dados os benefícios que constituem para a saúde.

No entanto, as algas podem apresentar também elevadas concentrações de elementos tóxicos, de entre os quais o arsénio (As) que, quando se encontra na sua forma inorgânica e em elevadas concentrações, pode ter efeitos prejudiciais para a saúde, podendo induzir carcinogénese do fígado, rim, bexiga e pele.

Como se pode constatar pela análise da literatura apresentada anteriormente, a concentração de arsénio total, orgânico e inorgânico não é consensual. Esta varia consoante o local de desenvolvimento das algas, bem como a sua capacidade para acumular metais pesados, dependente da biodisponibilidade destes na água circundante. Além disso, considera-se que a discrepância dos valores acima mencionados também pode ser justificada pelas diversas metodologias aplicadas por cada autor nas respetivas investigações. A confeção das algas pode 
ter efeito na quantidade de arsénio total, sendo que esta diminui como resultado deste processo.

Ao analisar, de forma pormenorizada, as concentrações de arsénio nas várias espécies de algas, é evidente que as castanhas são as que apresentam valores mais altos, comparativamente com as vermelhas. Uma vez que as algas Nori se enquadram nesta última categoria, a concentração deste elemento nas mesmas, encontra-se dentro do nível de ingestão diária tolerável estabelecido pela OMS $(0,146 \mathrm{mg} /$ dia para um peso corporal de $68 \mathrm{~kg}$ ). À semelhança de França, torna-se fulcral desenvolver um regulamento na Comunidade Europeia, que estipule o teor máximo de contaminantes presentes nas algas, uma vez que estas ainda não estão incluídas no Regulamento (CE) n. ${ }^{\circ}$ 466/2001 e podem constituir um potencial risco para a saúde humana.

\section{CONCLUSÕES}

Em suma, atribui-se elevada importância ao estudo da composição das algas marinhas comestíveis, nomeadamente da concentração de elementos essenciais e tóxicos, no sentido de avaliar potenciais efeitos na saúde da população.

Assim, conclui-se que as algas Nori quando consumidas com moderação (não excedendo as $12 \mathrm{~g} / \mathrm{dia}$ ) a concentração de arsénio inorgânico não é muito elevada, pelo que não é considerada tóxica para os consumidores.

Com o aumento significativo do consumo destas algas, considera-se importante adotar o exemplo de França em toda a Comunidade Europeia, estabelecendo um regulamento sobre o uso de algas marinhas para consumo humano.

\section{REFERÊNCIAS BIBLIOGRÁFICAS}

1. Higa T, Kuniyoshi M. Toxins associated with medicinal and edible seaweeds. $J$ Toxicol - Toxin Rev. 2000;19(2):119-37.

2. Desideri D, Cantaluppi C, Ceccotto F, Meli MA, Roselli C, Feduzi L. Essential and toxic elements in seaweeds for human consumption. J Toxicol Environ Heal - Part A Curr Issues. 2016;79(3):112-22.

3. Burtin P. Issn : 1579-4377 Nutritional Value of Seaweeds. Quality [Internet] 2003;2(4):498-503. Available from: http://ejeafche.uvigo.es/component/option,com_ docman/task,doc_download/gid,208/.

4. Brown EM, Allsopp PJ, Magee PJ, Gill Cl, Nitecki S, Strain CR, et al. Seaweed and human health. Nutr Rev. 2014;72(3):205-16.

5. European Food Safety Authority. Dietary exposure to inorganic arsenic in the European population. European Food Safety Authority Journal [Internet]. 2014 Mar;12(3). Available from: http://doi.wiley.com/10.2903/j.efsa.2014.3597.

6. Cheney D. Toxic and Harmful Seaweeds [Internet]. Seaweed in Health and Disease Prevention. Elsevier Inc.; 2016. 407-421 p. Available from: http://dx.doi.org/10.1016/ B978-0-12-802772-1.00013-0.

7. Noda $\mathrm{H}$. Health benefits and nutritional properties of nori. J Appl Phycol. 1993;5(2):255-8.

8. Zhao Y, Shang D, Ning J, Zhai Y. Arsenic and cadmium in the marine macroalgae (porphyra yezoensis and laminaria japonica)-forms and concentrations. Chem Speciat Bioavailab. 2012;24(3):197-203.

9. Mac Monagail M, Morrison L. Arsenic speciation in a variety of seaweeds and associated food products. Compr Anal Chem. 2019;85:267-310.

10. Zhao YF, Wu JF, Shang DR, Ning JS, Ding HY, Zhai YX. Arsenic species in edible seaweeds using in vitro biomimetic digestion determined by high-performance liquid chromatography inductively coupled plasma mass spectrometry. Int J Food Sci. $2014 ; 2014$.

11. Brandon EFA, Janssen PJCM, de Wit-Bos L. Arsenic: bioaccessibility from seaweed and rice, dietary exposure calculations and risk assessment. Food Addit Contam - Part A Chem Anal Control Expo Risk Assess. 2014;31(12):1993-2003.

12. WHO. Exposure to Arsenic: A Major Public Health Concern. Agriculture [Internet].
2010;5. Available from: http://www.who.int/ipcs/features/arsenic.pdf?ua=1. Acessed at: 05/03/2020

13. Rose M, Lewis J, Langford N, Baxter M, Origgi S, Barber M, et al. Arsenic in seaweedForms, concentration and dietary exposure. Food Chem Toxicol. 2007;45(7):1263-7. 14. International Agency for Research on Cancer(IARC). Arsenic and Arsenic Compounds Monograph. IARC Monogr Eval Carcinog Risks Humans [Internet]. 2012;100C:41-93. Available from: monographs.iarc.fr/ENG/Monographs/vol100C/ mono100C-6.pdf.

15. Taylor VF, Li Z, Sayarath V, Palys TJ, Morse KR, Scholz-Bright RA, et al. Distinct arsenic metabolites following seaweed consumption in humans. Sci Rep [Internet]. 2017;7(1):1-9. Available from: http://dx.doi.org/10.1038/s41598-017-03883-7.

16. García Sartal C, Barciela-Alonso M del C, Bermejo-Barrera P. Effect of the cooking procedure on the arsenic speciation in the bioavailable (dialyzable) fraction from seaweed. Microchem J [Internet]. 2012;105:65-71. Available from: http://dx.doi. org/10.1016/j.microc.2012.08.001.

17. Norman JA, Pickford CJ, Sanders TW, Waller M. Human intake of arsenic and iodine from seaweed-based food supplements and health foods available in the UK. Food Addit Contam. 1988;5(1):103-9.

18. Kumar MS, Sharma SA. Toxicological effects of marine seaweeds: a cautious insight for human consumption. Crit Rev Food Sci Nutr [Internet]. 2020;0(0):1-22. Available from: https://doi.org/10.1080/10408398.2020.1738334.

19. Hong DD, Hien HM, Son PN. Seaweeds from Vietnam used for functional food, medicine and biofertilizer. J Appl Phycol. 2007;19(6):817-26.

20. Taboada MC, Millán R, Miguez MI. Nutritional value of the marine algae wakame (Undaria pinnatifida) and nori (Porphyra purpurea) as food supplements. J Appl Phycol. 2013;25(5):1271-6.

21. Chen Q, Pan XD, Huang BF, Han JL. Distribution of metals and metalloids in dried seaweeds and health risk to population in southeastern China. Sci Rep [Internet]. 2018;8(1):1-7. Available from: http://dx.doi.org/10.1038/s41598-018-21732-z.

22. Almela C, Algora S, Benito V, Clemente MJ, Devesa V, Súñer MA, et al. Heavy metal, total arsenic, and inorganic arsenic contents of algae food products. J Agric Food Chem. 2002;50(4):918-23.

23. Ma Z, Lin L, Wu M, Yu H, Shang T, Zhang T, et al. Total and inorganic arsenic contents in seaweeds: Absorption, accumulation, transformation and toxicity. Aquaculture [Internet]. 2018;497:49-55. Available from: https://doi.org/10.1016/j. aquaculture.2018.07.040.

24. Bouga M, Combet E. Emergence of Seaweed and Seaweed-Containing Foods in the UK: Focus on Labeling, lodine Content, Toxicity and Nutrition. Foods. 2015;4(4):240-53.

25. Cheng JS, Li JH, Jiang HF, Ouyang XY. The solvent effect on the chemoselectivity of palladium-catalyzed oligomerization of 3, 3-dimethyl-1-butyne. Chinese Chem Lett. 2003;14(9):966-8.

26. Besada V, Andrade JM, Schultze F, González JJ. Heavy metals in edible seaweeds commercialised for human consumption. J Mar Syst [Internet]. 2009;75(1-2):305-13. Available from: http://dx.doi.org/10.1016/j.jmarsys.2008.10.010. 